\section{AB009. Bioprosthetics in airway surgery}

\section{Michael Lanuti}

Division of Thoracic Surgery, Massachusetts General Hospital, Boston, MA, USA

Correspondence to: Michael Lanuti, MD. Division of Thoracic Surgery, Massachusetts General Hospital, 55 Fruit Street, Founders 7, Boston, MA 02114, USA. Email: mlanuti@mgh.harvard.edu.

\begin{abstract}
Among many challenges in thoracic surgery, tracheal substitutes have been particularly elusive over nearly 75 years. Although nearly $4-5 \mathrm{~cm}$ of trachea can be resected and primarily reconstructed in properly selected patients, larger or more complicated resections have spawned innovation to overcome this limitation. The trachea functions as an airtight, mechanically stable passage from larynx to bronchi and facilitates clearance of secretions via a specialized epithelium. Ideal properties of a tracheal substitute include durable, non-immunogenic material that is resistant to infection and can easily incorporate surrounding tissue. Four methodologies have been implemented for airway reconstruction: autologous tissue, allotransplantation, bioprosthetics, and tissue engineering. Of note, complete circumferential prosthetic tracheal substitutes have historically failed. The most innovative solutions to tracheal replacement have been tissue engineering and bioprosthetics but both strategies have significant limitations. Tubularized aortic homograft supported by an endoluminal internal stent (Montgomery tube, silicone T-tube or nitinol stent) and external buttressing with muscle has been studied in patients with some limited success. A hypothesis has been put forward from some authors that aortic homografts contain undifferentiated stem cells that may play a role in migration, proliferation and cell signaling of host cells to form primitive tracheal elements including intima replacement with squamous or ciliated epithelium and chondrogenesis. In a few cases the internal stents were removed beyond 1-2 years with regeneration of cartilage elements within the graft. Despite this interesting biology, reported longterm complications include infection and tracheoesophageal fistula. Both aortic homograft and human acellular dermal matrix have experienced more success when implemented
\end{abstract}

as a patch repair of large non-circumferential airway defects. The etiology of these defects can be derived from trauma with loss of tissue domain, infection and erosion (such as tracheoesophageal fistulae), or partial resection for neoplasm. In the case of human acellular dermal matrix the bioprosthetic is meticulously sutured so that the dermal side faces the airway lumen. Since this tissue matrix normally is broken down by the host, it primary serves as a scaffold for tissue ingrowth. Of note, the luminal side of aortic homograft is also oriented to face the airway lumen and the wall is perforated with a 16 -gauge needle to promote tissue ingrowth and neovascularization. In the description of these reconstructions, repairs were routinely buttressed with vascularized tissue exteriorly and granulation tissue was observed at the site of reconstruction in many patients with mild tissue contracture and eventual native tissue ingrowth. It is understood that these patients require frequent surveillance bronchoscopy with debridement of granulation as necessary. Reported complications of this strategy were sputum retention, pneumonia and pneumomediastinum. One author reports selective use of hyperbaric oxygen in patients with complex repairs to enhance tissue ingrowth of the bioprosthetic. Tissue engineering generally involves the implantation of a biodegradable scaffold seeded with host cells that can repopulate the scaffold with new extracellular matrix. This has been reported in experimental settings with decellularized cadaveric trachea seeded with bone-marrow derived mesenchymal stroma cells as well as autologous respiratory epithelium. Initial attempts at tracheal tissue engineering showed promise in preclinical models but were associated with unacceptable outcomes in humans. Lastly, several studies have been published implementing three-dimensional (3D) printing technology to engineer tracheal substitutes that mimic mechanical behavior similar to native trachea. Biodegradable scaffolds of trachea have been $3 \mathrm{D}$ printed, some cultured with chondrocytes allowing formation of neocartilage, and tested in preclinical models. Although these investigations are very preliminary, $3 \mathrm{D}$ printing appears to provide infinite possibilities for tissue engineering.

Keywords: Trachea; bioprosthetics; homograft; tissue engineering; acellular dermis

doi: 10.21037/shc.2019.AB009

Cite this abstract as: Lanuti $M$. Bioprosthetics in airway surgery. Shanghai Chest 2019;3:AB009. 